\title{
Spatial Organization of the Ccq1-Tpz1-Poz1 Telomere Complex
}

\author{
Harry Scott ${ }^{1}$, Jin-Kwang $\mathrm{Kim}^{3}$, Clinton $\mathrm{Yu}^{4}$, Lan Huang ${ }^{4}$, Feng Qiao ${ }^{3}$, \& Derek J. Taylor ${ }^{1,2}$ \\ ${ }^{1}$ Departments of Pharmacology and ${ }^{2}$ Biochemistry Case Western Reserve University, Cleveland $\mathrm{OH}$ \\ 44106 \\ ${ }^{3}$ Departments of Biological Chemistry and ${ }^{4}$ Physiology and Biophysics, University of California Irvine, \\ Irvine CA 92697
}

\begin{abstract}
All linear chromosomes end in stretches of non-coding hexanucleotide repeats called telomeres [1]. The telomere exists to protect the genome and to facilitate healthy cellular growth. As one function, the ubiquitous telomere sequence absorbs the gradual loss in length that results from the inability of replicative DNA polymerases to extend the extreme ends of the lagging strand [4]. As such, the length of the telomere of a cell is an important determinant for correlating with life expectancy; where shorter telomeres generally indicate a shorter remaining lifespan [5]. Eventually, the telomeres become so short that a state of replicative senescence is induced to prevent further cell division and the potential loss of genetic information [6]. In highly proliferative cells, the enzyme called telomerase is expressed to attenuate this process of telomere erosion [7]. Germline cells and stem cells express telomerase, which synthesizes telomere DNA to avoid replicative senescence. Whereas telomerase activity is undetectable in healthy somatic cells, it is upregulated appreciably in $85-90 \%$ of all cancers [7]. This leads to telomere extension and replicative immortality, which allows the cancer cells to divide indefinitely. By improving our understanding of the processes involved in telomere homeostasis, it may be possible to develop novel methods to therapeutically target these processes in the context of cancer.
\end{abstract}

A group of specialized telomere proteins form a complex called shelterin that serves primarily to protect telomere DNA, but also functions as a recruitment platform for telomerase. While the molecular pathways of telomerase recruitment and telomere elongation are being uncovered, there are many gaps that remain in understanding important details of these processes. Most notably, it is unknown how the telomere-associated proteins interact to coordinate protection versus telomerase recruitment events. For example, in human telomeres, most shelterin proteins behave as negative regulators for telomerase, yet TPP1 positively regulates telomerase recruitment and activity $[8,9]$. While the precise interactions that govern protection and recruitment events are under investigation, the lack of structural data to correlate the current cell and biochemical data poses a major obstacle in understanding these critical processes.

Because of high conservation of composition and function among eukaryotic shelterins, the Schizosaccharomyces pombe telomeres are regulated in a way that is similar to the human system [10]. Telomere maintenance in $S$. pombe is regulated, in part, by physical interactions between the shelterin proteins Ccq1, Tpz1, and Poz1 [11]. Whereas interactions between Ccq1 and Tpz1 are known to facilitate telomerase recruitment through phosphorylated signaling of Ccq1 Thr93, the inclusion of Poz1 within the Ccq1-Tpz1-Poz1 (CTP) complex negatively impacts telomerase-mediated extension events [12]. Thus, it is critical to define the interactions between Ccq1, Tpz1 and Poz1 to understand the molecular switch that governs or prevents telomerase-mediated extension of telomeres.

We have used electron microscopy combined with single particle reconstruction techniques to determine the three-dimensional structure of the $S$. pombe CTP shelterin complex. Moreover, the localization and stoichiometry of each protein subunit within the CTP complex was identified using density labels and 
truncated protein constructs. These results indicate that the CTP complex is assembled from a dimer of trimers with two copies of Ccq1, Tpz1 and Poz1 proteins. Complementing our structural data, we used mass spectrometry and chemical fixation to identify protein-protein interactions within the complex at amino acid resolution. Using the combination of the MS and EM data, we report the first EM model of the CTP shelterin complex, recapitulating its structure in the non-extendable state.

\section{References:}

[1] Blackburn, E.H; Gall, J; Haven, N, J Mol Bio. 120 (1978), p. 33-53.

[2] Huffman, K; Levene, S; Wright, W, J Biol Chem. 275 (2000), p. 19719-19722.

[3] Wright, W; Tesmer, V; Shay J, Genes \& Development 11 (1997), p. 2801-2809.

[4] Blackburn, E; Greider, C; Szosttak, J, Nature Medicine 12 (2006), p. 1133-1138.

[5] Allsop, R; Vaziri, Harley, C, PNAS 89 (1992), p. 10114-8.

[6] Harley, C; Futcher, A; Greider, C, Nature 345 (1990), p. 458-60.

[7] Shay, J; Bacchetti, S, European Journal of Cancer 33 (1997), p. 787-791

[8] Xin, H; et al. Nature 445 (2007), p. 559-562.

[9] Wang, F; et al. Nature 445 (2007) p. 506-510

[10] de Lange, T, Genes \& Development 19 (2005), p. 2100-2110.

[11] Miyoshi, T; Kanoh, J; Saito, M; Ishikawa, F, Science 320 (2008), p. 1341-1344

[12] Jun, H; Liu, J; Feng Q, Genes \& Development 27 (2013), p. 1917-1931.

[13] The authors acknowledge funding from NIH (DP2 CA186571 to D.J.T. and R01 GM098943 to F.Q.) and the American Cancer Society (RSG-13-211-01-DMC to D.J.T.)

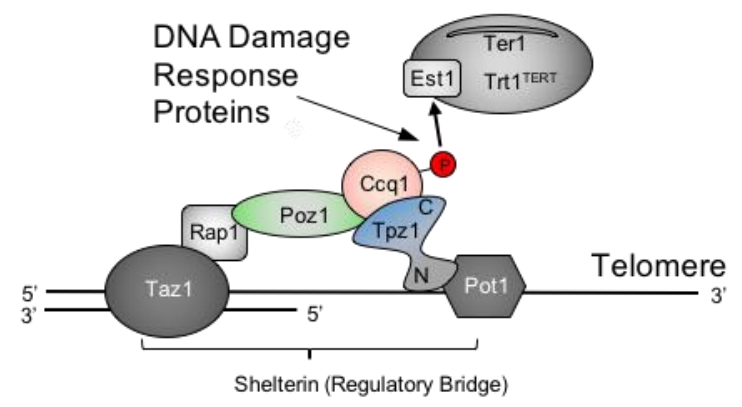

Figure 1. The CTP complex is responsible for modulating the telomere. Cellular events contribute to DNA damage response machinery phosphorylating Thr93 of Ccq1 leading to telomerase recruitment and telomere elongation.

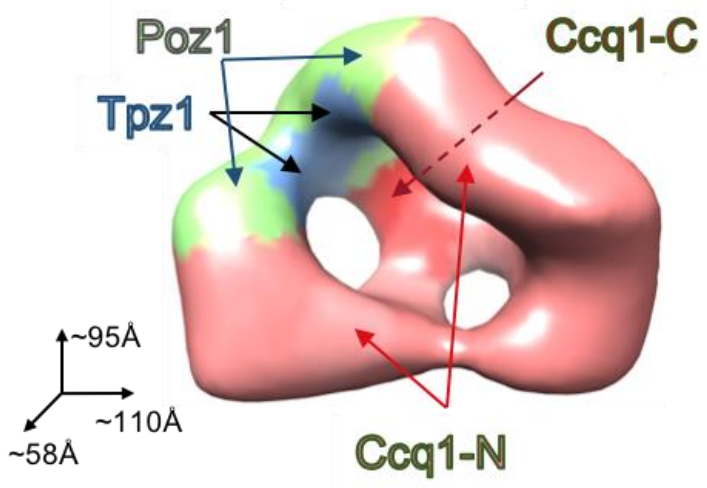

Figure 2. The CTP complex is a dimer of trimers. Salmon represents Ccq1, and red represents the Cterminal Ccq1 homodimerization region. Tpz1 (blue) bridges Ccq1 with Poz1 (green). 